Ephemeride von (56) Melete für die Opposition 1890.

$12^{\text {h }}$ M. Z. Berlin.

\begin{tabular}{|c|c|c|c|c|c|c|c|c|c|}
\hline 1890 & 1 & $a \mathrm{a}$ & $\mathrm{pp}$ & & & $\operatorname{app}$ & & $\log A$ & Ab.Zt \\
\hline Mai Io & $16^{\text {h }}$ & 15 & $m_{2} 8^{s} .03$ & & $10^{\circ}$ & $55^{\prime}$ & $6: 1$ & 0.076864 & $49^{\mathrm{m}} 54^{\mathrm{s}}$ \\
\hline I I & & 14 & $45 \cdot 34$ & & 10 & 47 & 34.6 & 0.075118 & 52 \\
\hline 12 & & I 4 & 1.56 & & 10 & 40 & 5.0 & 0.073450 & 49 \\
\hline I 3 & & 13 & 16.77 & & 10 & 32 & 37.8 & 0.071862 & 47 \\
\hline I 4 & & 12 & 31.04 & & 10 & 25 & 13.5 & 0.070356 & 45 \\
\hline 15 & & I I & 44.43 & & 10 & 17 & 52.6 & 0.068932 & 43 \\
\hline 16 & & 10 & 57.02 & & 10 & 10 & 35.6 & 0.067593 & 41 \\
\hline 17 & & 10 & 8.89 & & 10 & 3 & 22.8 & 0.066338 & 40 \\
\hline 18 & & 9 & 20.13 & & 9 & $5^{6}$ & I 4.8 & 0.065170 & 38 \\
\hline 19 & & 8 & 30.80 & & 9 & 49 & 12.0 & 0.064089 & 37 \\
\hline 20 & & 7 & 40.99 & & 9 & 42 & 15.0 & 0.063096 & 35 \\
\hline $2 \mathrm{I}$ & & 6 & 50.77 & & 9 & 35 & 24.2 & 0.062192 & 34 \\
\hline 22 & & 6 & 0.25 & & 9 & 28 & 40.0 & 0.061376 & 33 \\
\hline 23 & & 5 & $9.5^{\circ}$ & & 9 & 22 & 2.8 & 0.060649 & 32 \\
\hline 24 & & 4 & $18.6 \mathrm{I}$ & & 9 & I 5 & 33.2 & $0.0600 \mathrm{II}$ & $3 I$ \\
\hline 25 & & 3 & 27.65 & & 9 & 9 & I 1.5 & 0.059462 & 31 \\
\hline 26 & & 2 & $3^{6.7} \mathrm{I}$ & & 9 & 2 & $5^{8.1}$ & 0.059003 & $3^{\circ}$ \\
\hline 27 & & I & 45.88 & & 8 & $5^{6}$ & 53.6 & 0.058633 & $3^{\circ}$ \\
\hline 28 & 16 & 0 & 55.23 & & 8 & 50 & 58.0 & $0.05^{8} 35^{2}$ & $\begin{array}{l:ll}2 & 9 & 29\end{array}$ \\
\hline
\end{tabular}

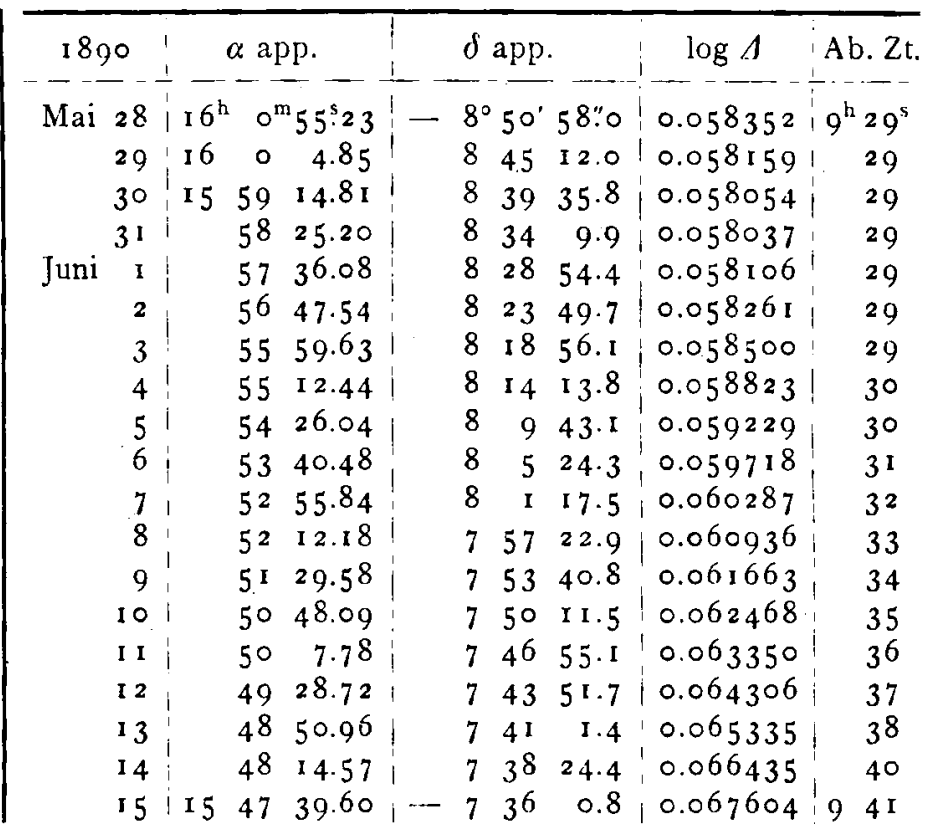

Düsseldorf I 890 Mai ro.

Opposition in AR. Mái 24. Grösse I O.I I.

Rob. Luther.

\title{
Sur la rotation du Soleil. $\left.{ }^{*}\right)$ \\ Par N.C. Dunćr.
}

(Note communiquée le I 2 Février I 890 .)

Pendant les étés de 1887, r 888 et 1889 , j'ai fait I arrangé mes observations de la sorte, que je n'aie pas eu des recherches sur la rotation du soleil, à l'aide d'un à faire que des mesures micrométriques entre des raies spectroscope à réseaux de diffraction de Rowland, appliqué spectrales dont les longueurs d'onde ne diffèrent que d'une au réfracteur de l'observatoire de Lund. Ce spectroscope unité d'Angström à peu près. C'est que j'ai mesuré, sur est d'une si grande puissance optique, qu' on peut, à son | des points opposés du bord solaire, les distances entre les aide, déterminer la différence en longueur d'onde de deux, raies spectrales suivantes, appartenantes au groupe $a$ du raies voisines du spectre solaire, avec une telle exactitude, spectre solaire: que l'erreur probable du résultat, obtenu pendant un été, n'atteigne pas 0.0000000002 millimètre. Par conséquent, on pourrail bien espérer d'obtenir, par une recherche soignée du déplacement des raies spectrales, sur des points opposés du bord solaire, des valeurs de la vitesse avec laquelle ces points s'approchent ou s'éloignent de la terre, assez exactes pour parvenir à la connaissance des lois de la rotation du soleil, même dans les latitudes héliocentriques élevées où l'on n'a jamais vu de tache. Je crois ne pas trop dire, en prétendant que cet espoir s'est réalisé. Je publierai autre part les détails complets de ces observations; ici, je n'en communique que les résultats.

Il me faut, cependant, dire quelques mots sur la méthode dont je me suis servi pour trouver le déplacement différences, exprimées en parties des tours de la vis microdes raies spectrales. Vu l'exactitude supérieure qu' on ob- métrique, qu' on obtient immédiatement par l'observation, tient en général par des observations différentielles, j'ai ! j'ai mesuré aussi les distances entre $\mathbf{I}$ raies, situées entre 
6252 et $633^{8}$ et dont les longueurs d'onde ont été très exactement déterminées par $M$. le prof. $H$. Rowland à Baltimore.

Les résultats de mes mesures sont donnés dans la table suivante où $\varphi$ est la latitude héliocentrique des points sur le bord solaire, $v$ la vitesse en kilometres avec laquelle le point sur le bord est se rapproche de la terre, e l'erreur probable de $v$, et $n$ le nombre des séries de mesures que j'ai prises dans les années différentes

$\begin{array}{rcccc}\varphi & v & e & n & \text { An. } \\ 0.3 & 1.90 & \pm 0.018 & 49 & 1888 \\ 0.4 & 2.05 & \pm 0.028 & 24 & 1889 \\ 0.6 & 1.99 & \pm 0.020 & 34 & 1887 \\ 14.9 & 1.85 & \pm 0.024 & 37 & 1887 \\ 15.0 & 1.92 & \pm 0.021 & 24 & 1889 \\ 15.2 & 1.77 & \pm 0.021 & 43 & 1888 \\ 29.9 & 1.55 & \pm 0.020 & 51 & 1888 \\ 30.0 & 1.56 & \pm 0.026 & 29 & 1887 \\ 30.1 & 1.64 & \pm 0.027 & 24 & 1889 \\ 44.9 & 1.16 & \pm 0.021 & 49 & 1888 \\ 44.9 & 1.19 & \pm 0.024 & 24 & 1889 \\ 45.3 & 1.21 & \pm 0.030 & 33 & 1887 \\ 59.9 & 0.70 & \pm 0.017 & 46 & 1888 \\ 60.0 & 0.77 & \pm 0.023 & 38 & 1887 \\ 60.0 & 0.74 & \pm 0.025 & 23 & 1889 \\ 74.7 & 0.32 & \pm 0.029 & 37 & 1887 \\ 74.8 & 0.39 & \pm 0.014 & 47 & 1888 \\ 75.0 & 0.32 & \pm 0.025 & 23 & 1889\end{array}$

Quand même les erreurs probables de ces moyennes ont des grandeurs assez différentes, je n'ai pas cru devoir les prendre en considération à la formation des positions normales, les erreurs systématiques dans les années particulières étant plus fortes que les erreurs accidentelles. En attribuant à toutes les moyennes le même poids, j'ai trouvé les suivantes valeurs normales de $v$, de $\xi \cos \varphi$ et de $\xi$, $\xi$ étant l'angle de rotation en 24 heures:

$\begin{array}{ccccc}\varphi & v & \xi \cos \varphi & \xi & n \\ 0.4 & 1.98 & 14.14 & 14.14 & 107 \\ 15.0 & 1.85 & 13.19 & 13.66 & 104 \\ 30.0 & 1.58 & 11.31 & 13.06 & 104 \\ 45.0 & 1.19 & 8.48 & 11.99 & 106 \\ 60.0 & 0.74 & 5.31 & 10.62 & 107 \\ 74.8 & 0.34 & 2.45 & 9.34 & 107\end{array}$

Ces valeurs de $\xi$, déduites des observations spectrosco piques, confirment assurément ce qu'avient révélé les observations sur les taches, à savoir que le temps de rotation des différentes zones de la surface solaire n'est pas le même, mais que la zone équatoriale fait un tour entier dans le temps le plus court, et que le temps de rotation augmente avec la latitude. Mais tandis que les taches qu' on a pu observer pendant plus d'une révolution, et qui par conséquent se prètent avec avantage aux recherches sur la rotation du soleil, ne se sont présentées que tout exceptionnellement au delà de $35^{\circ}$ de latitude et jamais au delà de $45^{\circ}$, et que la latitude la plus élevée où l'on a vu une tache n'atteigne pas les $55^{\circ}$, et que par conséquent on n'a jusqu' à présent su rien sur la rotation des deux calottes entre $55^{\circ}$ et les poles, mes observations étendent notre connaissance de ces faits jusqu' à la latitude de $75^{\circ}$, donc jusqu'au voisinage immédiat des poles.

J'ai employé les valeurs de $\xi \cos \varphi$ pour déduire des formules qui donnent les valeurs de l'angle de rotation en fonction de la latitude heliocentrique. On sait que M. Faye a, dans les recherches qu'il a faites sur ce sujet, adopté la forme

$$
\xi \cos \varphi=\left[a-b \sin \varphi^{2}\right] \cos \varphi \text {, }
$$

ou ce qui revient au même:

$$
\xi \cos \varphi=\left[a^{\prime}+b^{\prime} \cos \varphi^{2}\right] \cos \varphi,
$$

tandis que M. Spörer donne la préférence à la très simple forme

$$
\xi \cos \varphi=[a+b \cos \varphi] \cos \varphi
$$

déjà proposée par M. C. H. F. Peters. Mais en outre M. Spörer a employé la forme

ou bien

$$
\begin{aligned}
& \xi \cos \varphi=[a+b \cos \varphi+c \sin \varphi] \cos \varphi, \\
& \xi \cos \varphi=[a+b \cos \varphi] \cos \varphi+c^{\prime} \sin 2 \varphi .
\end{aligned}
$$

J'ai fait application de toutes ces trois formes aux valeurs de $\xi \cos \varphi$, fournies par mes observations, et j'ai trouvé, par la méthode des moindres carrés:

$\xi \cos \varphi=\left[14: 077-4.535 \sin \varphi^{2}\right] \cos \varphi$

$\xi \cos \varphi=\{7.286+6.723 \cos \varphi] \cos \varphi$

$\xi \cos \varphi=[8.596+5.522 \cos \varphi-0.759 \sin \varphi] \cos \varphi$

En comparant ces formules avec les observations, on obtient les erreurs résiduelles suivantes, $e^{\prime}, e^{\prime \prime}, e^{\prime \prime \prime}$.

$$
\begin{array}{cccc}
\varphi & e^{\prime} & e^{\prime \prime} & e^{\prime \prime \prime} \\
0.4 & +0.06 & +0.13 & +0.03 \\
15.0 & -0.12 & -0.12 & -0.07 \\
30.0 & +0.09 & -0.04 & +0.05 \\
45.0 & +0.12 & -0.03 & +0.02 \\
60.0 & -0.03 & -0.01 & -0.04 \\
74.8 & -0.13 & +0.08 & +0.01
\end{array}
$$

La somme des carrés de ces erreurs résiduelles est resp. $0.0583,0.0403$ et 0.0104 . L'accord de la formule (III) avec les observations est donc considérablement meilleur que celui des deux autres formules. Cependant, il faut avouer, que les erreurs résiduelles de la formule (II) et même celles de la formule (I) ne surpassent guère les erreurs probables des observations. La comparaison entre les observations spectroscopiques et les observations sur les taches montre que celles-la donnent une vitesse de rotation un peu plus petite que celles-ci. 